Article

\title{
Hypoalbuminemia Reflects Nutritional Risk, Body Composition and Systemic Inflammation and Is Independently Associated with Survival in Patients with Colorectal Cancer
}

\author{
Arwa S. Almasaudi 1,2,*, Ross D. Dolan ${ }^{3} \mathbb{D}$, Christine A. Edwards ${ }^{2} \mathbb{D}$ and Donald C. McMillan ${ }^{3}$ \\ 1 Department of Clinical Nutrition, Faculty of Applied Medical Science, King Abdulaziz University, \\ Jeddah 21589, Saudi Arabia \\ 2 Human Nutrition, School of Medicine, Dentistry and Nursing, College of Medical, \\ Veterinary and Life of Sciences, University of Glasgow, Glasgow Royal Infirmary, Glasgow G31 2ER, UK; \\ Christine.edwards@glasgow.ac.uk \\ 3 Academic Unit of Surgery, College of Medical, Veterinary and Life of Sciences-University of Glasgow, \\ Royal Infirmary, Glasgow G31 2ER, UK; \\ Ross.Dolan@glasgow.ac.uk (R.D.D.); Donald.McMillan@glasgow.ac.uk (D.C.M.) \\ * Correspondence: a.almasaudi.1@research.gla.ac.uk or aalmasaudi@kau.edu.sa
}

Received: 10 June 2020; Accepted: 15 July 2020; Published: 21 July 2020

check for updates

\begin{abstract}
It has long been recognized that albumin has prognostic value in patients with cancer. However, although the Global Leadership Initiative on Malnutrition GLIM criteria (based on five diagnostic criteria, three phenotypic criteria and two etiologic criteria) recognize inflammation as an important etiologic factor in malnutrition, there are limited data regarding the association between albumin, nutritional risk, body composition and systemic inflammation, and whether albumin is associated with mortality independent of these parameters. The aim of this study was to examine the relationship between albumin, nutritional risk, body composition, systemic inflammation, and outcomes in patients with colorectal cancer (CRC). A retrospective cohort study $(n=795)$ was carried out in which patients were divided into normal and hypoalbuminaemic groups (albumin $<35 \mathrm{~g} / \mathrm{L}$ ) in the presence and absence of a systemic inflammatory response C-reactive protein (CRP $>10$ and $<10 \mathrm{mg} / \mathrm{L}$, respectively). Post-operative complications, severity of complications and mortality were considered as outcome measures. Categorical variables were analyzed using Chi-square test $\chi^{2}$ or linear-by-linear association. Survival data were analyzed using univariate and multivariate Cox regression. In the presence of a systemic inflammatory response, hypoalbuminemia was directly associated with Malnutrition Universal Screening Tool MUST $(p<0.001)$ and inversely associated with Body Mass Index BMI $(p<0.001)$, subcutaneous adiposity $(p<0.01)$, visceral obesity $(p<0.01)$, skeletal muscle index $(p<0.001)$ and skeletal muscle density $(p<0.001)$. There was no significant association between hypoalbuminemia and either the presence of complications or their severity. In the absence of a systemic inflammatory response $(n=589)$, hypoalbuminemia was directly associated with MUST $(p<0.05)$ and inversely associated with BMI $(p<0.01)$, subcutaneous adiposity $(p<0.05)$, visceral adiposity $(p<0.05)$, skeletal muscle index $(p<0.01)$ and skeletal muscle density $(p<0.001)$. Hypoalbuminemia was, independently of inflammatory markers, associated with poorer cancer-specific and overall survival (both $p<0.001$ ). The results suggest that hypoalbuminemia in patients with $\mathrm{CRC}$ reflects both increased nutritional risk and greater systemic inflammatory response and was independently associated with poorer survival in patients with CRC.
\end{abstract}

Keywords: colorectal cancer; albumin; body composition; nutrition status; systemic inflammatory response and colorectal cancer outcomes 


\section{Introduction}

Albumin is the most abundant plasma protein in humans, representing approximately $50 \%$ of the total protein content of plasma. Albumin has a myriad of important physiologic functions that are essential for normal health. In an adult, the normal range of serum albumin has been defined as $35-50 \mathrm{~g} / \mathrm{L}$ and concentrations $<35 \mathrm{~g} / \mathrm{L}$ have been termed hypoalbuminemia [1]. The presence of hypoalbuminemia has long been associated with adverse clinical outcomes across a number of clinical specialties. For example, in a study of a large number of patients $(n=204,819)$ undergoing surgery, mainly for cancer (56\%), Meyer et al. showed that hypoalbuminemia was associated with post-operative complications, increased length of hospital stay, and mortality. They recently concluded that there was a need to assess preoperative albumin levels and to manage nutritional status accordingly [2]. Some have concluded that this is antiquated thinking and ignores the fact that albumin is a negative acute phase reactant and that cancer can be considered as an inflammatory condition [3,4], and this may in part explain the prognostic value of albumin in patients with cancer. Also, serum albumin concentrations do not change in response to short-term changes in nutrient intake and in states of malnutrition [5-7].

In addition, it is clear that systemic inflammation reduces albumin concentrations independent of nutrient intake [5]. Indeed, the reduction in circulating albumin concentrations, as well as an increase in C-reactive protein, are linked with poor outcomes in patients with colorectal cancer independent of tumor stage and host factors [8-10]. Therefore, despite being addressed repeatedly in the literature, there is still a lack of quantitative data regarding the clinical significance of the factors that are associated with hypoalbuminemia (including nutritional risk, body composition and systemic inflammatory markers). Furthermore, little is known about whether hypoalbuminemia is associated with survival independent of these parameters. Through the use of Computerized tomography (CT scans), body composition can now be assessed as part of the routine clinical work up of patients with cancer, in particular in patients with operable disease [11]. The aim of the present study was to examine the relationship between hypoalbuminemia, nutrition-related risk, body composition, systemic inflammation and mortality in patients with operable colorectal cancer.

\section{Patients and Methods}

\subsection{Patients}

A retrospective cohort study from a prospective database of patients undergoing surgery for colorectal cancer (prospectively maintained as part of a surgical audit) was carried out. Consecutive patients who underwent potentially curative resection for colorectal cancer between March 2013 and June 2016 at Glasgow Royal Infirmary were studied. All patients with recorded routine laboratory measurements of white cells, neutrophil, lymphocyte, monocyte and platelet counts, albumin and C-reactive protein, and who had a preoperative CT scan, were included in the analysis. Patient comorbidity was classified using the American Society of Anesthesiologists (ASA) grading system [12]. Patients were classified as having anemia based on World Health Organization (WHO) guidelines for males (hemoglobin $(\mathrm{Hb})<130 \mathrm{~g} / \mathrm{L}$ and females; $\mathrm{Hb}<120 \mathrm{~g} / \mathrm{L}$ ) [13]. Patients were divided into normal and hypoalbuminemic groups according to the widely accepted definition of hypoalbuminemia (serum albumin $>35 /<35 \mathrm{~g} / \mathrm{L}$ ) [1]. A subgroup analysis for those patients without a systemic inflammatory response (CRP $<10 \mathrm{mg} / \mathrm{L} ; n=589)$ has been carried out to examine whether there is an independent effect of hypoalbuminemia on MUST and nutritional status based on BMI and body composition parameters. Any uncertainties of clinical outcome were addressed by a review of electronic and/or the physical case report.

The study had ethical approval in the UK (West of Scotland Ethics Committee: GN170N474) and was conducted in accordance with the Declaration of Helsinki. Furthermore, the study conformed to the Strengthening The Reporting of OBservational Studies in Epidemiolog (STROBE) guidelines for cohort studies. 


\subsection{Nutritional Risk}

As part of routine clinical practice in the UK, MUST scores were recorded in patient notes within $24 \mathrm{~h}$ of admission. Clinical staff carried out the assessment (usually nursing staff).

MUST incorporates three components to determine the overall risk for malnutrition: current weight status using BMI, unintentional weight loss, and acute disease effect that has induced a phase of nil by mouth for $>5$ days. Each parameter can be rated as 0,1 , or 2 . Overall risk for malnutrition is established as low (score $=0)$, medium (score $=1)$, or high $($ score $\geq 2)$ [14].

\subsection{Body Composition}

CT images were obtained at the level of the third lumbar vertebra. Patients whose scans were taken 3 months or more prior to their surgery were excluded from the study. The median and range for the interval between CT scanning and operation was 0.91 months (0.03-2.83) prior to surgery. Scans with significant movement artefact or missing regions of interest were not considered for inclusion. Each image was analyzed using ImageJ software [15].

Region of interest (ROI) measurements were made of visceral fat (VFA), subcutaneous fat (SFA) and skeletal muscle areas (SMA) $\left(\mathrm{cm}^{2}\right)$ using standard Hounsfield Unit (HU) ranges (adipose tissue -190 to -30 , and skeletal muscle -29 to +150$)$. These were then normalized for height ${ }^{2}$ to create indices; total fat index (TFI, $\mathrm{cm}^{2} / \mathrm{m}^{2}$ ), subcutaneous fat index (SFI, $\mathrm{cm}^{2} / \mathrm{m}^{2}$ ), visceral fat index $\left(\mathrm{VFI}, \mathrm{cm}^{2} / \mathrm{m}^{2}\right)$, and skeletal muscle index (SMI, $\left.\mathrm{cm}^{2} / \mathrm{m}^{2}\right)$. Skeletal muscle radiodensity (SMD, HU) was measured from the same ROI used to calculate SMI, as its mean HU.

As part of an ongoing research program in the Academic Unit of Surgery, training for body composition measurements and analysis was provided. The CT scan analysis was carried out by clinical research fellows (AA and RD). The inter-rater reliability was assessed in a sample of 40 patient images using interclass correlation coefficients (ICCC) (TFA ICCC $=1.000$, SFA ICCC $=1.000$, VFA ICCC $=1.000$, SMA ICCC $=0.998$, SMD ICCC $=0.972$ ).

On the basis of CT-derived body composition, there have been a number of threshold values associated with survival in patients with cancer. The thresholds used for SMI and SMD were derived from large patient cohorts [16,17]. In particular, SMI is recognized to have prognostic value; the threshold used was derived from a large cohort of patients with operable colorectal cancer [16].

Visceral obesity was defined as VFA $>160 \mathrm{~cm}^{2}$ for male patients and $>80 \mathrm{~cm}^{2}$ for female patients [18]. High subcutaneous fat index (SFI) was defined as $>50.0 \mathrm{~cm}^{2} / \mathrm{m}^{2}$ in males and $>42.0 \mathrm{~cm}^{2} / \mathrm{m}^{2}$ in females [19]. Sarcopenia was described by Caan and colleagues as SMI $<52.3 \mathrm{~cm}^{2} / \mathrm{m}^{2}$ if BMI $<30 \mathrm{~kg} / \mathrm{m}^{2}$ and $\mathrm{SMI}<54.3 \mathrm{~cm}^{2} / \mathrm{m}^{2}$ if BMI $>30 \mathrm{~kg} / \mathrm{m}^{2}$ in male patients and $\mathrm{SMI}<38.6 \mathrm{~cm}^{2} / \mathrm{m}^{2}$ if BMI $<30 \mathrm{~kg} / \mathrm{m}^{2}$ and an SMI $<46.6 \mathrm{~cm}^{2} / \mathrm{m}^{2}$ if BMI $>30 \mathrm{~kg} / \mathrm{m}^{2}$ in female patients [16]. Myosteatosis was defined by $\mathrm{SMD}<41 \mathrm{HU}$ in patients with $\mathrm{BMI}<25 \mathrm{~kg} / \mathrm{m}^{2}$ and $<33 \mathrm{HU}$ in patients with $\mathrm{BMI}>25 \mathrm{~kg} / \mathrm{m}^{2}$ [17].

\subsection{Inflammatory Markers}

Prior to surgery, there was routine clinical assessment of the systemic inflammatory response using C-reactive protein, albumin and a differential white cell count. The clinical chemistry laboratory at Glasgow Royal Infirmary participated in external quality assurance/proficiency testing programs. Performance was acceptable throughout, which indicated that any changes in assay methodology did not result in any bias. An autoanalyzer was used to measure serum CRP $(\mathrm{mg} / \mathrm{L})$ and albumin $(\mathrm{g} / \mathrm{L})$ concentrations Grouping of the variables CRP, albumin, white cell, neutrophil, lymphocyte, monocyte and platelet counts was carried out using standard thresholds [20-23]. 


\subsection{Statistical Analysis}

Statistical analysis was performed using SPSS software, version 24.0 [24]. The data were manually extracted from the prospective dataset into SPSS. Categorical variables were analyzed using chi-square test $\chi 2$ test for linear-by-linear association, or $\chi 2$ test for 2-by-2 tables. Missing data were excluded from the analysis on a variable by variable basis.

Those with mortality within 30 days of the index procedure or during the index admission were excluded from subsequent survival analysis. The time between the date of surgery and the date of death of any cause was used to define overall survival (OS). The time between the date of surgery and the date of death of cancer was used to define cancer specific survival (CS). The rationale for including both survival end points was that nutritional biomarkers may impact on both end points. Survival data were analyzed using univariate and multivariate Cox regression. Those variables associated to a degree of $p<0.1$ were entered into a backward conditional multivariate model. Two tailed $p$ values $<0.001$ were considered statistically significant.

\subsection{Results}

A total of 795 patients were included having undergone potentially curative surgery for colorectal cancer. The majority were male (55\%) and less than 75 years old $(74 \%)$, had low comorbidity $(68 \%)$, were not anemic $(60 \%)$, had a MUST score of $0(79 \%)$ and were overweight or obese $(66 \%)$. The majority of patients also had subcutaneous obesity $(82 \%)$, visceral obesity $(73 \%)$, low SMI $(53 \%)$ and low SMD $(63 \%)$ and did not have an elevated systemic inflammatory response as evidenced by white cell count $(70 \%)$, neutrophil count $(89 \%)$, monocyte count $(88 \%)$, platelet count $(88 \%)$ and C-reactive protein $(75 \%)$.

The relationships between hypoalbuminemia and clinical and pathological characteristics such as body composition and host systemic inflammatory response are shown in Table 1.

Table 1. The relationship between hypoalbuminemia, clinicopathological, CT-derived body composition and systemic inflammatory response in 795 patients undergoing surgery for colorectal cancer.

\begin{tabular}{|c|c|c|c|c|}
\hline \multirow{2}{*}{\multicolumn{2}{|c|}{ Characteristic }} & \multicolumn{3}{|c|}{ Total $n=795$} \\
\hline & & $\begin{array}{c}\text { Albumin }>35 \mathrm{~g} / \mathrm{L} \\
n=545(\%)\end{array}$ & $\begin{array}{c}\text { Albumin }<35 \mathrm{~g} / \mathrm{L} \\
n=250(\%)\end{array}$ & $p$-Value ${ }^{\mathrm{a}, \mathrm{b}}$ \\
\hline \multicolumn{5}{|c|}{ Clinicopathological } \\
\hline \multirow{3}{*}{ Age } & $<65 y$ & $221(41)$ & $64(25)$ & \multirow{3}{*}{$<0.001$} \\
\hline & $65-74 y$ & $225(41)$ & $79(32)$ & \\
\hline & $>74 \mathrm{y}$ & $99(18)$ & $107(43)$ & \\
\hline \multirow{2}{*}{ Sex } & Male & $302(55)$ & $138(55)$ & \multirow{2}{*}{0.955} \\
\hline & Female & $243(45)$ & $112(45)$ & \\
\hline \multirow{4}{*}{$\mathrm{ASA}^{\mathrm{c}}$} & 1 & $113(25)$ & $46(18)$ & \multirow{4}{*}{0.004} \\
\hline & 2 & $241(45)$ & $108(44)$ & \\
\hline & 3 & $147(28)$ & $78(32)$ & \\
\hline & 4 & $11(2)$ & $14(6)$ & \\
\hline \multirow{5}{*}{$\mathrm{TNM}^{\mathrm{d}}$} & 0 & $15(3)$ & $3(1)$ & \multirow{5}{*}{0.001} \\
\hline & 1 & $147(28)$ & $29(12)$ & \\
\hline & 2 & $176(33)$ & $125(50)$ & \\
\hline & 3 & $184(34)$ & $75(30)$ & \\
\hline & 4 & $12(2)$ & $16(7)$ & \\
\hline
\end{tabular}


Table 1. Cont.

\begin{tabular}{|c|c|c|c|c|}
\hline \multirow{2}{*}{\multicolumn{2}{|c|}{ Characteristic }} & \multicolumn{3}{|c|}{ Total $n=795$} \\
\hline & & $\begin{array}{l}\text { Albumin }>35 \mathrm{~g} / \mathrm{L} \\
n=545(\%)\end{array}$ & $\begin{array}{c}\text { Albumin }<35 \mathrm{~g} / \mathrm{L} \\
n=250(\%)\end{array}$ & $p$-Value ${ }^{a, b}$ \\
\hline \multicolumn{5}{|c|}{ Nutrition Status and Body Composition } \\
\hline \multirow{2}{*}{ Hemoglobin } & Normal & $389(72)$ & $82(33)$ & \multirow{2}{*}{$<0.001$} \\
\hline & Anemia & $155(28)$ & $165(67)$ & \\
\hline \multirow{4}{*}{ BMI $\left(\mathrm{kg} / \mathrm{m}^{2}\right)$} & Underweight & $22(4)$ & $16(7)$ & \multirow{4}{*}{$<0.001$} \\
\hline & Normal weight & $142(27)$ & $99(40)$ & \\
\hline & Overweight & $177(33)$ & $76(31)$ & \\
\hline & Obese & $191(36)$ & $54(22)$ & \\
\hline \multirow{3}{*}{ MUST score ${ }^{\mathrm{e}}$} & 0 & $217(86)$ & $59(61)$ & \multirow{3}{*}{$<0.001$} \\
\hline & 1 & $13(5)$ & $18(19)$ & \\
\hline & 2 & $23(9)$ & $20(21)$ & \\
\hline \multirow{2}{*}{$\begin{array}{l}\text { Subcutaneous } \\
\text { adiposity }\end{array}$} & No & $79(16)$ & $53(24)$ & \multirow{2}{*}{0.010} \\
\hline & Yes & $421(84)$ & $170(76)$ & \\
\hline \multirow{2}{*}{ Visceral obesity } & No & $125(24)$ & $77(33)$ & \multirow{2}{*}{0.013} \\
\hline & Yes & $397(76)$ & $159(67)$ & \\
\hline \multirow{2}{*}{ Low $\mathrm{SMI}^{\mathrm{f}}$} & No & $264(53)$ & $75(34)$ & \multirow{2}{*}{$<0.001$} \\
\hline & Yes & $236(47)$ & $148(66)$ & \\
\hline \multirow{2}{*}{ Low SMD ${ }^{g}$} & No & $223(44)$ & $49(21)$ & \multirow{2}{*}{$<0.001$} \\
\hline & Yes & $288(56)$ & $183(79)$ & \\
\hline \multicolumn{5}{|c|}{ Systemic Inflammatory Response } \\
\hline \multirow{3}{*}{ White cell count } & $<8.5 \times 10^{9-1}$ & $406(75)$ & $153(62)$ & \multirow{3}{*}{$<0.001$} \\
\hline & $8.5-11 \times 10^{9-1}$ & $106(20)$ & $60(24)$ & \\
\hline & $>11 \times 10^{9-1}$ & $31(6)$ & $34(14)$ & \\
\hline \multirow{3}{*}{ Lymphocyte count } & $>3.0 \times 10^{9-1}$ & $29(5)$ & $7(3)$ & \multirow{3}{*}{0.074} \\
\hline & $1.0-3.0 \times 10^{9-1}$ & $450(83)$ & $205(82)$ & \\
\hline & $<1.0 \times 10^{9-1}$ & $66(12)$ & $38(15)$ & \\
\hline \multirow{2}{*}{ Neutrophil count } & $<7.5 \times 10^{9-1}$ & $506(93)$ & $205(82)$ & \multirow{2}{*}{$<0.001$} \\
\hline & $>7.5 \times 10^{9-1}$ & $39(7)$ & $45(18)$ & \\
\hline \multirow{2}{*}{ Monocyte count } & $<0.9 \times 10^{9-1}$ & $495(92)$ & $205(84)$ & \multirow{2}{*}{0.002} \\
\hline & $>0.9 \times 10^{9-1}$ & $46(8)$ & $39(16)$ & \\
\hline \multirow{2}{*}{ Platelet count } & $<400 \times 10^{9-1}$ & $506(93)$ & $193(78)$ & \multirow{2}{*}{$<0.001$} \\
\hline & $>400 \times 10^{9-1}$ & $36(7)$ & $54(22)$ & \\
\hline \multirow{2}{*}{$\mathrm{CRP}^{\mathrm{h}}$} & $<10 \mathrm{mg} / \mathrm{L}$ & $461(85)$ & $128(51)$ & 0001 \\
\hline & $>10 \mathrm{mg} / \mathrm{L}$ & 79 (15) & $121(49)$ & $<0.001$ \\
\hline
\end{tabular}


Table 1. Cont.

\begin{tabular}{|c|c|c|c|c|}
\hline \multirow{2}{*}{\multicolumn{2}{|c|}{ Characteristic }} & \multicolumn{3}{|c|}{ Total $n=795$} \\
\hline & & \multirow{2}{*}{$\begin{array}{c}\text { Albumin }>35 \mathrm{~g} / \mathrm{L} \\
n=545(\%)\end{array}$} & \multirow[t]{2}{*}{$\begin{array}{c}\text { Albumin }<35 \mathrm{~g} / \mathrm{L} \\
n=250(\%)\end{array}$} & \multirow[t]{2}{*}{$p$-Value ${ }^{\mathrm{a}, \mathrm{b}}$} \\
\hline & & & & \\
\hline \multirow{2}{*}{$\begin{array}{l}\text { Non-infective } \\
\text { complication }\end{array}$} & No & $447(83)$ & $191(78)$ & \multirow{2}{*}{0.068} \\
\hline & Yes & $95(18)$ & $55(22)$ & \\
\hline \multirow{2}{*}{$\begin{array}{c}\text { Infective } \\
\text { complication }\end{array}$} & No & $394(73)$ & $168(68)$ & \multirow{2}{*}{0.206} \\
\hline & Yes & $148(27)$ & $78(32)$ & \\
\hline \multirow{3}{*}{ Clavien Dindo grade } & 0 & $337(62)$ & $142(58)$ & \multirow{3}{*}{0.309} \\
\hline & $1-2$ & $154(28)$ & $80(32)$ & \\
\hline & $3-5$ & $50(9)$ & $24(10)$ & \\
\hline \multirow{3}{*}{ 5-year survival } & Alive & $432(81)$ & $140(57)$ & \multirow{3}{*}{$<0.001$} \\
\hline & Cancer-specific death & $57(11)$ & $66(27)$ & \\
\hline & Non-cancer death & $45(8)$ & $38(16)$ & \\
\hline \multicolumn{5}{|c|}{$\begin{array}{l}\text { a Chi test for linear by linear association; }{ }^{\mathrm{b}} \text { Significant values in bold } p<0.05{ }^{\mathrm{c}} \text { ASA: American Society of } \\
\text { Anesthesiologists; }{ }^{\mathrm{d}} \text { TNM: Tumor-Node-Metastasis; }{ }^{\mathrm{e}} \text { MUST: Malnutrition Universal Screening Tool. Overall risk } \\
\text { for malnutrition is established as low }(\text { score }=0) \text {, medium (score }=1) \text {, or high }(\text { score } \geq 2) ;{ }^{\mathrm{f}} \text { SMI: Skeletal Muscle } \\
\text { Index; }{ }^{\mathrm{g}} \text { SMD: Skeletal Muscle Density; }{ }^{\mathrm{h}} \text { CRP: C-Reactive Protein. } \\
\text { In the presence of a systemic inflammatory response }(n=795) \text {, hypoalbuminemia was } \\
\text { directly associated with greater age }(p<0.001) \text {, anemia }(p<0.001) \text {, comorbidity }(p<0.01) \\
\text { Tumor-Node-Metastasis TNM }(p<0.001) \text { and MUST }(p<0.001) \text { and inversely associated with } \\
\text { BMI }(p<0.001) \text {, subcutaneous adiposity }(p<0.01) \text {, visceral obesity }(p<0.01) \text {, skeletal muscle index } \\
(p<0.001) \text { and skeletal muscle density }(p<0.001) \text { and all components of a differential white cell count } \\
\text { (all } p<0.001) \text { except lymphocyte count }(p=0.074) \text {. There was no significant association between } \\
\text { hypoalbuminemia and either the presence of complications or their severity. Hypoalbuminemia was } \\
\text { directly associated with greater risk of cancer and non-cancer deaths }(p<0.001) \text {. } \\
\text { The relationships between hypoalbuminemia, clinical and pathological characteristics, and body } \\
\text { composition are shown in Table } 2 \text {. }\end{array}$} \\
\hline \multicolumn{5}{|c|}{$\begin{array}{ll} & \text { Total } n=589 \\
\end{array}$} \\
\hline \multicolumn{2}{|c|}{ Characteristic } & $\begin{array}{c}\text { Albumin }>35 \mathrm{~g} / \mathrm{L} \\
n=461(\%)\end{array}$ & $\begin{array}{c}\text { Albumin }<35 \mathrm{~g} / \mathrm{L} \\
n=128(\%)\end{array}$ & $p$-Value ${ }^{\mathrm{a}, \mathrm{b}}$ \\
\hline \multicolumn{5}{|c|}{ Clinicopathological } \\
\hline \multirow{3}{*}{ Age } & $<65 \mathrm{y}$ & $185(40)$ & $31(24)$ & \multirow{3}{*}{$<0.001$} \\
\hline & $65-74$ y & $187((41)$ & $42(33)$ & \\
\hline & $>74 y$ & 89 (19) & $55(43)$ & \\
\hline \multirow{2}{*}{ Sex } & Male & $256(55)$ & $70(55)$ & \multirow{2}{*}{0.865} \\
\hline & Female & $205(45)$ & $58(45)$ & \\
\hline \multirow{4}{*}{$\mathrm{ASA}^{\mathrm{c}}$} & 1 & $118(26)$ & $30(24)$ & \multirow{4}{*}{0.094} \\
\hline & 2 & $201(45)$ & $49(40)$ & \\
\hline & 3 & $124(27)$ & $39(31)$ & \\
\hline & 4 & $8(2)$ & $7(6)$ & \\
\hline
\end{tabular}


Table 2. Cont.

\begin{tabular}{|c|c|c|c|c|}
\hline \multirow{2}{*}{\multicolumn{2}{|c|}{ Characteristic }} & \multicolumn{3}{|c|}{ Total $n=589$} \\
\hline & & $\begin{array}{c}\text { Albumin }>35 \mathrm{~g} / \mathrm{L} \\
n=461(\%)\end{array}$ & $\begin{array}{c}\text { Albumin }<35 \mathrm{~g} / \mathrm{L} \\
n=128(\%)\end{array}$ & $p$-Value ${ }^{a, b}$ \\
\hline \multirow{5}{*}{$\mathrm{TNM}^{\mathrm{d}}$} & 0 & $14(3)$ & $2(2)$ & \multirow{5}{*}{0.024} \\
\hline & 1 & $132(29)$ & $22(17)$ & \\
\hline & 2 & $142(32)$ & $54(43)$ & \\
\hline & 3 & $151(34)$ & $42(33)$ & \\
\hline & 4 & $11(2)$ & $7(5)$ & \\
\hline \multicolumn{5}{|c|}{ Nutrition Status and Body Composition } \\
\hline \multirow{2}{*}{ Hemoglobin } & Normal & $332(72)$ & $48(38)$ & \multirow{2}{*}{$<0.001$} \\
\hline & Anemia & $128(28)$ & $77(62)$ & \\
\hline \multirow{4}{*}{$\mathrm{BMI}\left(\mathrm{kg} / \mathrm{m}^{2}\right)$} & Underweight & $18(4)$ & $5(4)$ & \multirow{4}{*}{0.009} \\
\hline & Normal weight & $121(27)$ & $52(41)$ & \\
\hline & Over weight & $157(35)$ & $37(29)$ & \\
\hline & Obese & $154(34)$ & $32(25)$ & \\
\hline \multirow[b]{2}{*}{ MUST score ${ }^{\mathrm{e}}$} & 0: No risk & $186(84)$ & $36(69)$ & \multirow[b]{2}{*}{0.016} \\
\hline & $\begin{array}{c}1 \text { and 2: medium and } \\
\text { high risk }\end{array}$ & $36(16)$ & $16(31)$ & \\
\hline \multirow{2}{*}{$\begin{array}{l}\text { Subcutaneous } \\
\text { adiposity }\end{array}$} & No & $64(15)$ & $28(25)$ & \multirow{2}{*}{0.016} \\
\hline & Yes & $362(85)$ & $86(75)$ & \\
\hline \multirow{2}{*}{ Visceral obesity } & No & $104(23)$ & $39(33)$ & \multirow{2}{*}{0.036} \\
\hline & Yes & $341(77)$ & $80(67)$ & \\
\hline \multirow{2}{*}{ Low SMI ${ }^{\mathrm{f}}$} & No & $227(53)$ & $44(37)$ & \multirow{2}{*}{0.005} \\
\hline & Yes & $199(47)$ & $70(61)$ & \\
\hline \multirow{2}{*}{ Low SMD $g$} & No & $192(44)$ & $32(27)$ & \multirow{2}{*}{0.001} \\
\hline & Yes & $243(56)$ & $85(73)$ & \\
\hline \multicolumn{5}{|c|}{ Systemic Inflammatory Response } \\
\hline \multirow{3}{*}{ White cell count } & $<8.5 \times 10^{9-1}$ & $354(77)$ & $94(75)$ & \multirow{3}{*}{0.853} \\
\hline & $8.5-11 \times 10^{9-1}$ & $86(19)$ & $27(22)$ & \\
\hline & $>11 \times 10^{9-1}$ & $19(4)$ & $4(3)$ & \\
\hline \multirow{3}{*}{ Lymphocyte count } & $>3.0 \times 10^{9-1}$ & $26(6)$ & $3(2)$ & \multirow{3}{*}{0.079} \\
\hline & $1.0-3.0 \times 10^{9-1}$ & $378(82)$ & $104(81)$ & \\
\hline & $<1.0 \times 10^{9-1}$ & $57(12)$ & $21(16)$ & \\
\hline \multirow{2}{*}{ Neutrophil count } & $<7.5 \times 10^{9-1}$ & $434(94)$ & $116(91)$ & \multirow{2}{*}{0.157} \\
\hline & $>7.5 \times 10^{9-1}$ & $27(6)$ & $12(9)$ & \\
\hline \multirow{2}{*}{ Monocyte count } & $<0.9 \times 10^{9-1}$ & $424(93)$ & $112(90)$ & \multirow{2}{*}{0.243} \\
\hline & $>0.9 \times 10^{9-1}$ & $33(7)$ & $13(10)$ & \\
\hline Plotolotont & $<400 \times 10^{9-1}$ & $434(95)$ & $116(92)$ & \\
\hline Platelet count & $>400 \times 10^{9-1}$ & $24(5)$ & $10(8)$ & 0.253 \\
\hline
\end{tabular}


Table 2. Cont.

\begin{tabular}{|c|c|c|c|c|}
\hline \multirow{2}{*}{\multicolumn{2}{|c|}{ Characteristic }} & \multicolumn{3}{|c|}{ Total $n=589$} \\
\hline & & \multirow{2}{*}{$\begin{array}{c}\text { Albumin }>35 \mathrm{~g} / \mathrm{L} \\
n=461(\%)\end{array}$} & \multirow[t]{2}{*}{$\begin{array}{c}\text { Albumin }<35 \mathrm{~g} / \mathrm{L} \\
n=128(\%)\end{array}$} & \multirow[t]{2}{*}{$p$-Value ${ }^{\mathrm{a}, \mathrm{b}}$} \\
\hline & & & & \\
\hline \multirow{2}{*}{$\begin{array}{l}\text { Non-infective } \\
\text { complication }\end{array}$} & No & $381(83)$ & $98(78)$ & \multirow{2}{*}{0.162} \\
\hline & Yes & 77 (17) & $28(22)$ & \\
\hline \multirow{2}{*}{$\begin{array}{c}\text { Infective } \\
\text { complication }\end{array}$} & No & $337(74)$ & $90(71)$ & \multirow{2}{*}{0.630} \\
\hline & Yes & $121(26)$ & $36(29)$ & \\
\hline \multirow{3}{*}{ Clavien Dindo grade } & 0 & $287(63)$ & $76(60)$ & \multirow{3}{*}{0.707} \\
\hline & $1-2$ & $122(27)$ & $37(29)$ & \\
\hline & $3-5$ & $46(10)$ & $13(11)$ & \\
\hline \multirow{3}{*}{ 5-year survival } & Alive & $369(80)$ & $79(62)$ & \multirow{3}{*}{$<0.001$} \\
\hline & Cancer-specific death & $52(11)$ & $31(24)$ & \\
\hline & Non-cancer death & $40(9)$ & $18(14)$ & \\
\hline \multicolumn{5}{|c|}{$\begin{array}{l}{ }^{a} \text { Chi test for linear by linear association; }{ }^{b} \text { Significant values in bold } p<0.05 ;{ }^{c} \text { ASA: American Society of } \\
\text { Anesthesiologists; }{ }^{d} \text { TNM: Tumor-Node-Metastasis; }{ }^{\text {e }} \text { MUST: Malnutrition Universal Screening Tool. Overall risk } \\
\text { for malnutrition is established as low (score }=0) \text {, medium (score }=1) \text {, or high (score } \geq 2) ;{ }^{f} \text { SMI: Skeletal Muscle Index; } \\
\text { g SMD: Skeletal Muscle Density.In the absence of a systemic inflammatory response (C-reactive protein }<10 \mathrm{mg} / \mathrm{L} \text {, } \\
n=589) \text {, hypoalbuminemia was directly associated with greater age }(p<0.001) \text {, anemia }(p<0.001), \text { TNM }(p<0.05) \\
\text { and MUST }(p<0.05) \text { and inversely associated with BMI }(p<0.01) \text {, subcutaneous adiposity }(p<0.05) \text {, visceral adiposity } \\
(p<0.05) \text {, skeletal muscle index }(p<0.01) \text { and skeletal muscle density }(p<0.001), \text { but not components of a differential } \\
\text { white cell count. There was also no significant association between hypoalbuminemia and either the presence of } \\
\text { complications or their severity. Hypoalbuminemia was directly associated with greater risk of cancer and non-cancer } \\
\text { deaths }(p<0.001) \text {. }\end{array}$} \\
\hline
\end{tabular}

\subsection{Survival}

The relationship between hypoalbuminemia, nutrition status, body composition and cancer-specific survival in patients undergoing surgery for colorectal cancer is shown in Table 3.

Table 3. The relationship between hypoalbuminemia, nutritional risk, CT-derived body composition and cancer-specific survival in 795 patients undergoing surgery for colorectal cancer.

\begin{tabular}{|c|c|c|c|c|}
\hline \multirow[b]{2}{*}{ Variable $^{a}$} & \multicolumn{4}{|c|}{ Cancer-Specific Survival } \\
\hline & $\begin{array}{l}\text { Univariate } \\
\text { HR }(95 \% \mathrm{CI})^{*}\end{array}$ & $p$-Value & $\begin{array}{l}\text { Multivariate } \\
\text { HR (95\% CI) }\end{array}$ & $p$-Value \\
\hline Age $(<65 / 65-74 />74)$ y & $1.35(1.07-1.70)$ & 0.010 & $1.05(0.69-1.62)$ & 0.152 \\
\hline ASA grade $^{\mathrm{b}}(1 / 2 / 3 / 4)$ & $1.29(1.03-1.63)$ & 0.025 & $1.31(0.86-1.98)$ & 0.145 \\
\hline TNM stage $(0 / 1 / 2 / 3 / 4)$ & $2.52(1.98-3.20)$ & $<0.001$ & $3.42(2.16-5.40)$ & $<0.001$ \\
\hline Hypoalbuminemia (no/yes) & $2.93(2.06-4.18)$ & $<0.001$ & $1.68(0.84-3.35)$ & 0.059 \\
\hline Anemia (no/yes) & $1.64(1.15-2.34)$ & 0.006 & $1.24(0.65-2.35)$ & 0.192 \\
\hline $\begin{array}{c}\mathrm{BMI}\left(\mathrm{kg} / \mathrm{m}^{2}\right) \\
\text { (underweight/normal/overweight/obese) }\end{array}$ & $0.81(0.66-0.99)$ & 0.042 & $0.88(0.61-1.26)$ & 0.588 \\
\hline MUST score $^{c}(0 / 1 / 2)$ & $1.79(1.27-2.51)$ & 0.001 & $1.96(1.38-2.77)$ & $<0.001$ \\
\hline Subcutaneous adiposity (no/yes) & $0.79(0.49-1.26)$ & 0.328 & - & - \\
\hline
\end{tabular}


Table 3. Cont.

\begin{tabular}{|c|c|c|c|c|}
\hline \multirow{2}{*}{ Variable $^{a}$} & \multicolumn{4}{|c|}{ Cancer-Specific Survival } \\
\hline & $\begin{array}{c}\text { Univariate } \\
\text { HR }(95 \% \text { CI })^{*}\end{array}$ & $p$-Value & $\begin{array}{l}\text { Multivariate } \\
\text { HR }(95 \% \text { CI) }\end{array}$ & $p$-Value \\
\hline Visceral obesity (no/yes) & $0.94(0.62-1.43)$ & 0.796 & - & - \\
\hline Low SMI ${ }^{d}$ (no/yes) & $1.20(0.82-1.80)$ & 0.326 & - & - \\
\hline Low SMD ${ }^{c}$ (no/yes) & $1.38(0.92-2.07)$ & 0.118 & - & - \\
\hline
\end{tabular}

On univariate analysis, age $(p<0.010)$, ASA $(p<0.05)$, TNM stage $(p<0.001)$, albumin $(p<0.001)$, anemia $(p<0.01)$, MUST score $(p<0.001)$ and BMI $(p<0.05)$ were significantly associated with cancer-specific survival. On multivariate analysis, TNM stage (HR $=3.42 ; 95 \%$ CI 2.16-5.40, $p<0.001$ ) and MUST score (HR $=1.96 ; 95 \%$ CI $1.38-2.77, p=0.007)$ were independently associated with cancer-specific survival. The relationships between hypoalbuminemia, systemic inflammation and cancer-specific survival in patients undergoing surgery for colorectal cancer is shown in Table 4.

Table 4. The relationship between hypoalbuminemia, systemic inflammatory response and cancer-specific survival in 795 patients undergoing surgery for colorectal cancer.

\begin{tabular}{|c|c|c|c|c|}
\hline \multirow[b]{2}{*}{ Variable $^{a}$} & \multicolumn{4}{|c|}{ Cancer-Specific Survival } \\
\hline & $\begin{array}{c}\text { Univariate } \\
\text { HR }(95 \% \text { CI })^{*}\end{array}$ & $p$-Value & $\begin{array}{l}\text { Multivariate } \\
\text { HR (95\% CI) }\end{array}$ & $p$-Value \\
\hline Age $(<65 / 65-74 />74)$ y & $1.35(1.07-1.70)$ & 0.010 & $1.14(0.89-1.46)$ & 0.164 \\
\hline ASA grade $(1 / 2 / 3 / 4)$ & $1.29(1.03-1.63)$ & 0.025 & $1.21(0.95-1.55)$ & 0.072 \\
\hline TNM stage $(0 / 1 / 2 / 3 / 4)$ & $2.52(1.98-3.20)$ & $<0.001$ & $2.37(1.86-3.01)$ & $<0.001$ \\
\hline Hypoalbuminemia (no/yes) & $2.93(2.06-4.18)$ & $<0.001$ & $2.58(1.78-3.73)$ & $<0.001$ \\
\hline CRP (normal/abnormal) & $1.94(1.34-2.80)$ & $<0.001$ & $1.21(0.79-1.84)$ & 0.442 \\
\hline White cell count (normal/abnormal) & $1.55(1.21-1.99)$ & $<0.001$ & $1.03(0.71-1.50)$ & 0.719 \\
\hline Neutrophil count (normal/abnormal) & $2.62(1.69-4.08)$ & $<0.001$ & $1.79(1.11-2.87)$ & 0.016 \\
\hline Monocyte count (normal/abnormal) & $2.36(1.50-3.73)$ & $<0.001$ & $1.67(1.04-2.70)$ & 0.167 \\
\hline Platelet count (normal/abnormal) & $1.69(1.05-2.74)$ & 0.030 & $0.85(0.48-1.49)$ & 0.944 \\
\hline
\end{tabular}

On univariate analysis, albumin $(p<0.001)$ and all inflammatory markers $(p<0.05)$ were significantly associated with overall survival. On multivariate analysis, TNM stage (HR = 2.37; 95\% CI 1.86-3.01, $p=<0.001)$, albumin (HR $=2.58 ; 95 \%$ CI 1.78-3.73, $p \leq 0.001)$ and neutrophil count (HR $=1.79 ; 95 \%$ CI 1.11-2.87, $p=0.016)$ were independently associated with cancer-specific survival.

The relationship between hypoalbuminemia, nutrition status, body composition and overall survival in patients undergoing surgery for colorectal cancer is shown in Table 5. 
Table 5. The relationship between hypoalbuminemia, nutritional risk, CT-derived body composition and overall survival in 795 patients undergoing surgery for colorectal cancer.

\begin{tabular}{|c|c|c|c|c|}
\hline \multirow{2}{*}{ Variable $^{a}$} & \multicolumn{4}{|c|}{ Overall Survival } \\
\hline & $\begin{array}{l}\text { Univariate HR } \\
(95 \% \mathrm{CI})^{*}\end{array}$ & $p$-Value & $\begin{array}{c}\text { Multivariate HR } \\
(95 \% \mathrm{CI})\end{array}$ & $p$-Value \\
\hline Age $(<65 / 65-74 />74)$ y & $1.63(1.36-1.95)$ & $<0.001$ & $1.65(1.20-2.26)$ & 0.002 \\
\hline $\operatorname{ASA}_{\text {grade }}{ }^{\mathrm{b}}(1 / 2 / 3 / 4)$ & $1.61(1.35-1.93)$ & $<0.001$ & $1.39(0.99-1.93)$ & 0.093 \\
\hline TNM stage $(0 / 1 / 2 / 3 / 4)$ & $1.77(1.48-2.11)$ & $<0.001$ & $2.16(1.56-3.01)$ & $<0.001$ \\
\hline Hypoalbuminemia (no/yes) & $2.62(1.99-3.45)$ & $<0.001$ & $1.52(0.87-2.66)$ & 0.112 \\
\hline Anemia (no/yes) & $1.74(1.32-2.30)$ & $<0.001$ & $1.16(0.69-1.94)$ & 0.290 \\
\hline $\begin{array}{c}\mathrm{BMI}\left(\mathrm{kg} / \mathrm{m}^{2}\right) \\
\text { (underweight/normal/overweight/obese) }\end{array}$ & $0.77(0.66-0.90)$ & 0.001 & $0.87(0.65-1.16)$ & 0.627 \\
\hline MUST score ${ }^{c}(0 / 1 / 2)$ & $1.63(1.24-2.14)$ & $<0.001$ & $1.53(1.14-2.05)$ & 0.004 \\
\hline Subcutaneous adiposity (no/yes) & $0.66(0.47-0.94)$ & 0.021 & - & - \\
\hline Visceral obesity (no/yes) & $0.87(0.63-1.18)$ & 0.377 & - & - \\
\hline Low SMI d (no/yes) & $1.6(1.19-2.23)$ & 0.002 & - & - \\
\hline Low SMD ${ }^{c}$ (no/yes) & $1.79(1.30-2.48)$ & $<0.001$ & $1.14(0.60-2.15)$ & 0.629 \\
\hline
\end{tabular}

${ }^{*}$ HR: Hazard ratio, CI: Confidence Interval a Variables associated to a degree of $p<0.1$ were entered into a backward conditional multivariate model. Two-tailed $p$ values $<0.001$ were considered statistically significant; ${ }^{b}$ ASA: American Society of Anesthesiologists; ${ }^{\mathrm{c}}$ MUST: Malnutrition Universal Screening Tool. Overall risk for malnutrition is

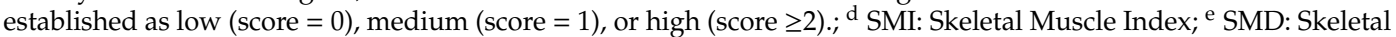
Muscle Density.

On univariate analysis, age $(p<0.00)$, ASA $(p<0.001)$, TNM $(p<0.001)$, albumin $(p<0.001)$, anemia $(p<0.001)$, BMI $(p<0.001)$, MUST score $(p<0.001)$, subcutaneous adiposity $(p<0.050 .021)$, SMI $(p=0.002)$ and SMD $(p<0.001)$ were significantly associated with overall survival. On multivariate analysis, age $(\mathrm{HR}=1.65 ; 95 \%$ CI 1.20-2.26, $p=0.002)$, TNM $(\mathrm{HR}=2.16$; 95\% CI 1.56-3.01, $p<0.001)$ and MUST score $(\mathrm{HR}=1.53 ; 95 \%$ CI $1.14-2.05, p=0.004)$, were independently associated with overall survival.

The relationship between hypoalbuminemia, systemic inflammation and overall survival in patients undergoing surgery for colorectal cancer is shown in Table 6.

Table 6. The relationship between hypoalbuminemia, systemic inflammation, and overall survival in in 795 patients undergoing surgery for colorectal cancer.

\begin{tabular}{ccccc}
\hline \multirow{2}{*}{ Variable $^{\text {a }}$} & \multicolumn{4}{c}{ Overall Survival } \\
\cline { 2 - 5 } & $\begin{array}{c}\text { Univariate } \\
\text { HR }(\mathbf{9 5 \%} \text { CI) }\end{array}$ & $p$-Value & $\begin{array}{c}\text { Multivariate } \\
\text { HR (95\% CI) }\end{array}$ & $p$-Value \\
\hline Age $(<65 / 65-74 />74)$ y & $1.63(1.36-1.95)$ & $<\mathbf{0 . 0 0 1}$ & $1.33(1.09-1.61)$ & $\mathbf{0 . 0 0 4}$ \\
\hline ASA grade $(1 / 2 / 3 / 4)$ & $1.61(1.35-1.93)$ & $<\mathbf{0 . 0 0 1}$ & $1.43(1.18-1.72)$ & $<\mathbf{0 . 0 0 1}$ \\
\hline TNM stage $(0 / 1 / 2 / 3 / 4)$ & $1.77(1.48-2.11)$ & $<\mathbf{0 . 0 0 1}$ & $1.72(1.43-2.07)$ & $<\mathbf{0 . 0 0 1}$ \\
\hline Hypoalbuminemia (no/yes) & $2.62(1.99-3.45)$ & $<\mathbf{0 . 0 0 1}$ & $1.95(1.45-2.62)$ & $<\mathbf{0 . 0 0 1}$ \\
\hline CRP (normal/abnormal) & $1.85(1.39-2.46)$ & $<\mathbf{0 . 0 0 1}$ & $1.167(0.83-1.62)$ & 0.318 \\
\hline White cell count (normal/abnormal) & $1.53(1.26-1.87)$ & $<\mathbf{0 . 0 0 1}$ & $0.98(0.73-1.32)$ & 0.897 \\
\hline Neutrophil count (normal/abnormal) & $2.29(1.60-3.28)$ & $<\mathbf{0 . 0 0 1}$ & $1.59(1.08-2.33)$ & $\mathbf{0 . 0 1 7}$ \\
\hline Monocyte count (normal/abnormal) & $2.19(1.52-3.14)$ & $<\mathbf{0 . 0 0 1}$ & $1.57(1.07-2.29)$ & $\mathbf{0 . 0 1 9}$ \\
\hline Platelet count (normal/abnormal) & $1.73(1.19-2.52)$ & $\mathbf{0 . 0 0 4}$ & $1.05(0.69-1.61)$ & 0.628 \\
\hline
\end{tabular}

${ }^{*}$ HR: Hazard ratio, CI: Confidence Interval a Variables associated to a degree of $p<0.1$ were entered into a backward conditional multivariate model. Two-tailed $p$ values $<0.001$ were considered statistically significant. 
On univariate analysis, albumin $(p<0.001)$ and all inflammatory markers $(p<0.01)$ were significantly associated with overall survival. On multivariate analysis, age $(\mathrm{HR}=1.33$; 95\% CI 1.09-1.61, $p<0.01$ ), ASA (HR $=1.43 ; 95 \%$ CI 1.18-1.72, $p<0.001)$, TNM stage (HR = 1.72; 95\% CI 1.43-2.07, $p<0.001$ ), albumin (HR $=1.95 ; 95 \%$ CI 1.45-2.62, $p<0.001)$, neutrophil count $(\mathrm{HR}=1.59 ; 95 \% \mathrm{CI} 1.08-2.33, p<0.05)$ and monocyte count $(\mathrm{HR}=1.57,95 \% \mathrm{CI} 1.07-2.29, p<0.05)$ were independently associated with overall survival.

The Kaplan-Meier curves in Figure 1 show the relationship between albumin (normal vs. low) and cancer-specific survival ( $\log \operatorname{rank} p=0.001$ ), and Figure 2 shows the relationship between albumin (normal vs. low) and overall survival (log rank $p=0.001)$.

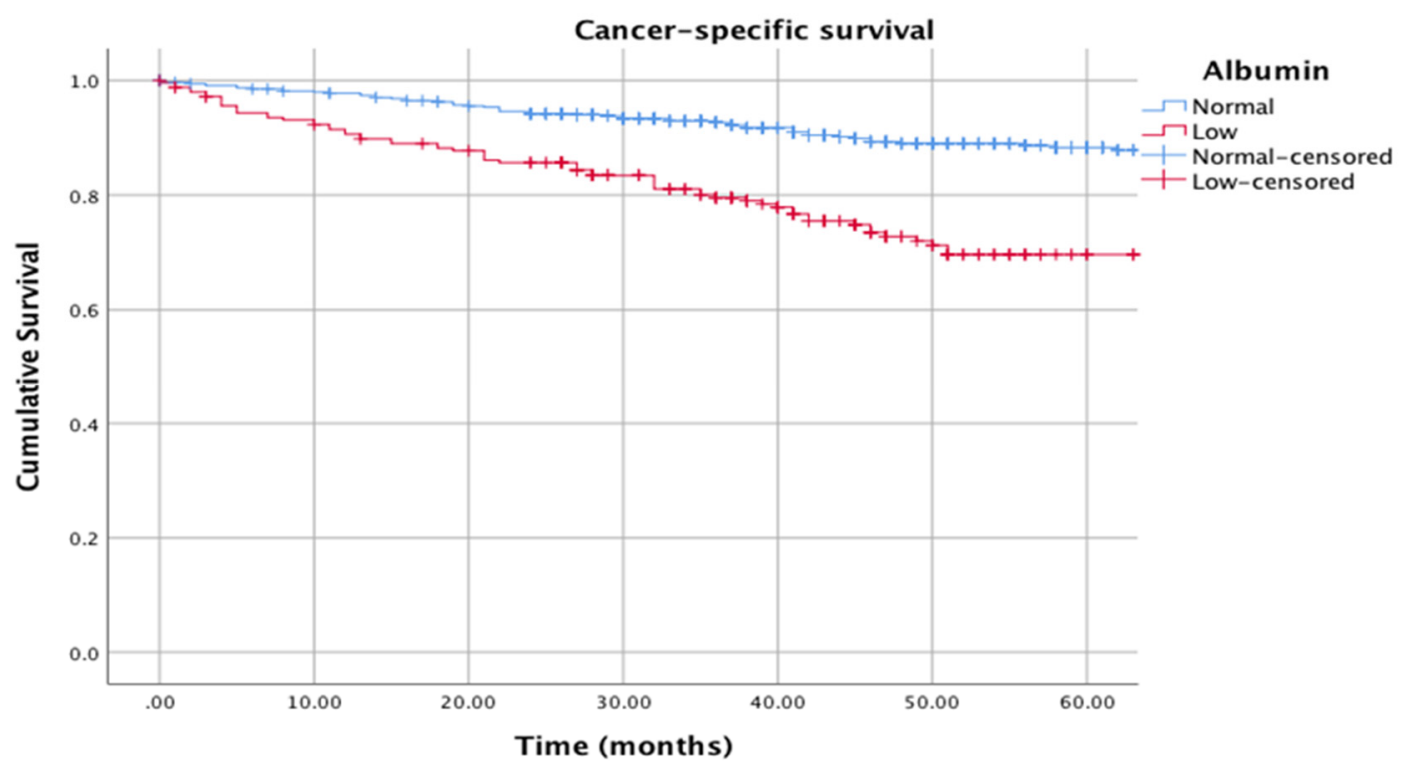

Figure 1. Kaplan-Meier curves showing the relationship between albumin (normal: $>35 \mathrm{~g} / \mathrm{L}$ vs. low: $<35 \mathrm{~g} / \mathrm{L}$ ) and cancer-specific survival. $n=795$. Median follow-up: 38 months. $\log$ rank $p=0.001$.

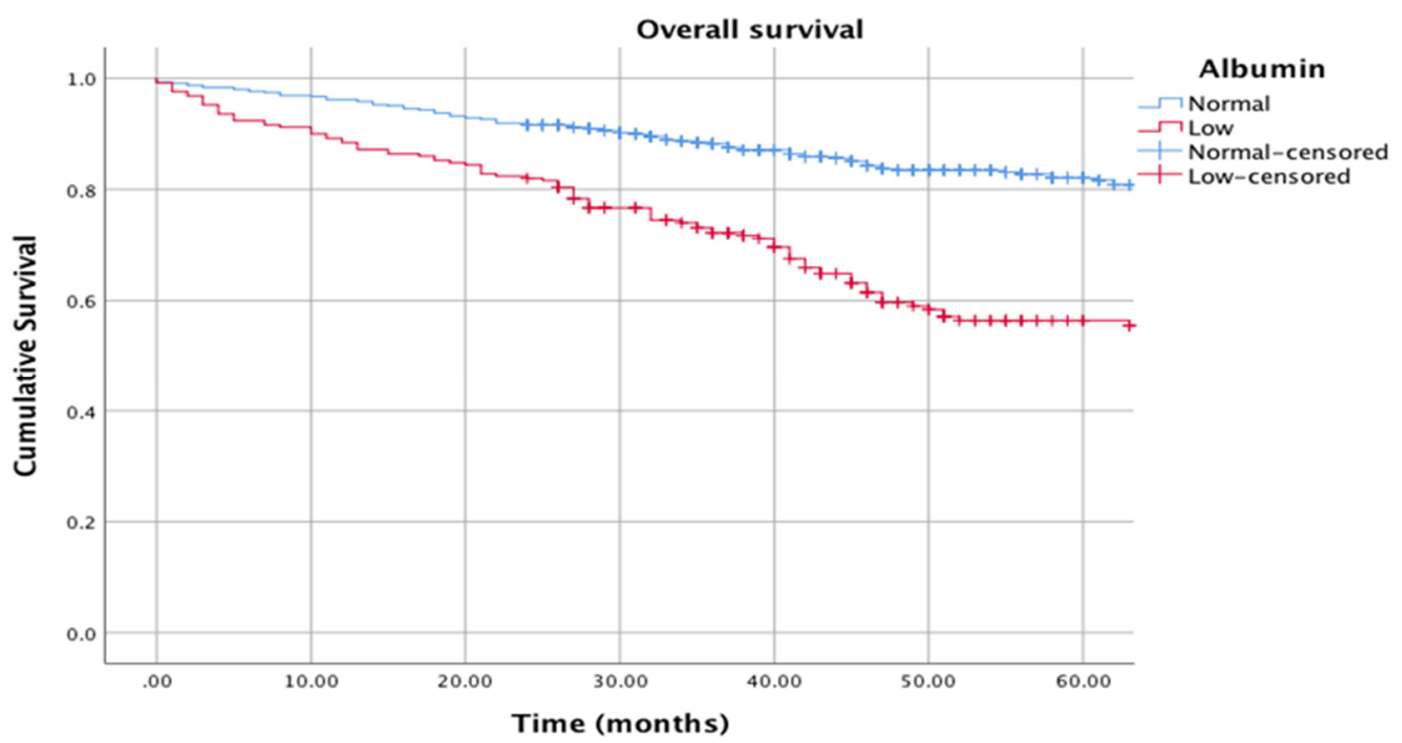

Figure 2. Kaplan-Meier curves showing the relationship between albumin (normal: $>35 \mathrm{~g} / \mathrm{L}$ vs. low: $<35 \mathrm{~g} / \mathrm{L}$ ) and overall survival. $n=795$. Median follow-up: 38 months. $\log \operatorname{rank} p=0.001$.

Figure 3 shows the relationship between albumin (normal: $>35 \mathrm{~g} / \mathrm{L}$ vs. low: $<35 \mathrm{~g} / \mathrm{L}$ ) and cancer-specific survival (in the absence of a systemic inflammatory response, $\mathrm{CRP}<10 \mathrm{mg} / \mathrm{L})(\log$ rank 
$p=0.001$ ), and Figure 4 shows the relationship between albumin (normal: $>35 \mathrm{~g} / \mathrm{L}$ vs. low: $<35 \mathrm{~g} / \mathrm{L}$ ) and overall survival (in the absence of a systemic inflammatory response, $\mathrm{CRP}<10 \mathrm{mg} / \mathrm{L})(\log$ rank $p=0.001)$.

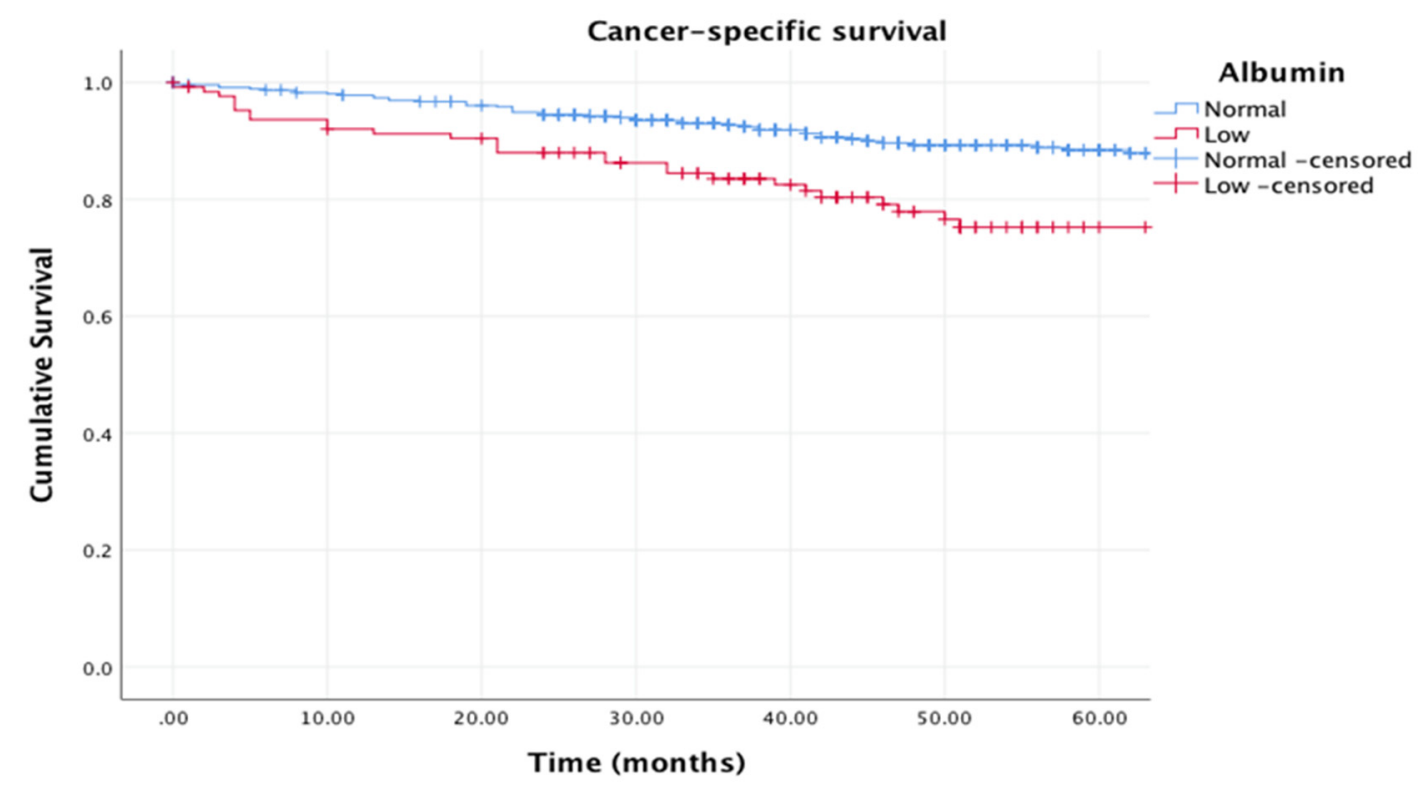

Figure 3. Kaplan-Meier curves showing the relationship between albumin (normal: $>35 \mathrm{~g} / \mathrm{L}$ vs. low: $<35 \mathrm{~g} / \mathrm{L}$ ) and cancer-specific survival (in the absence of a systemic inflammatory response, $\mathrm{CRP}<10 \mathrm{mg} / \mathrm{L}$ ). $n=589$. Median follow-up: 38 months. Log rank $p=0.001$.

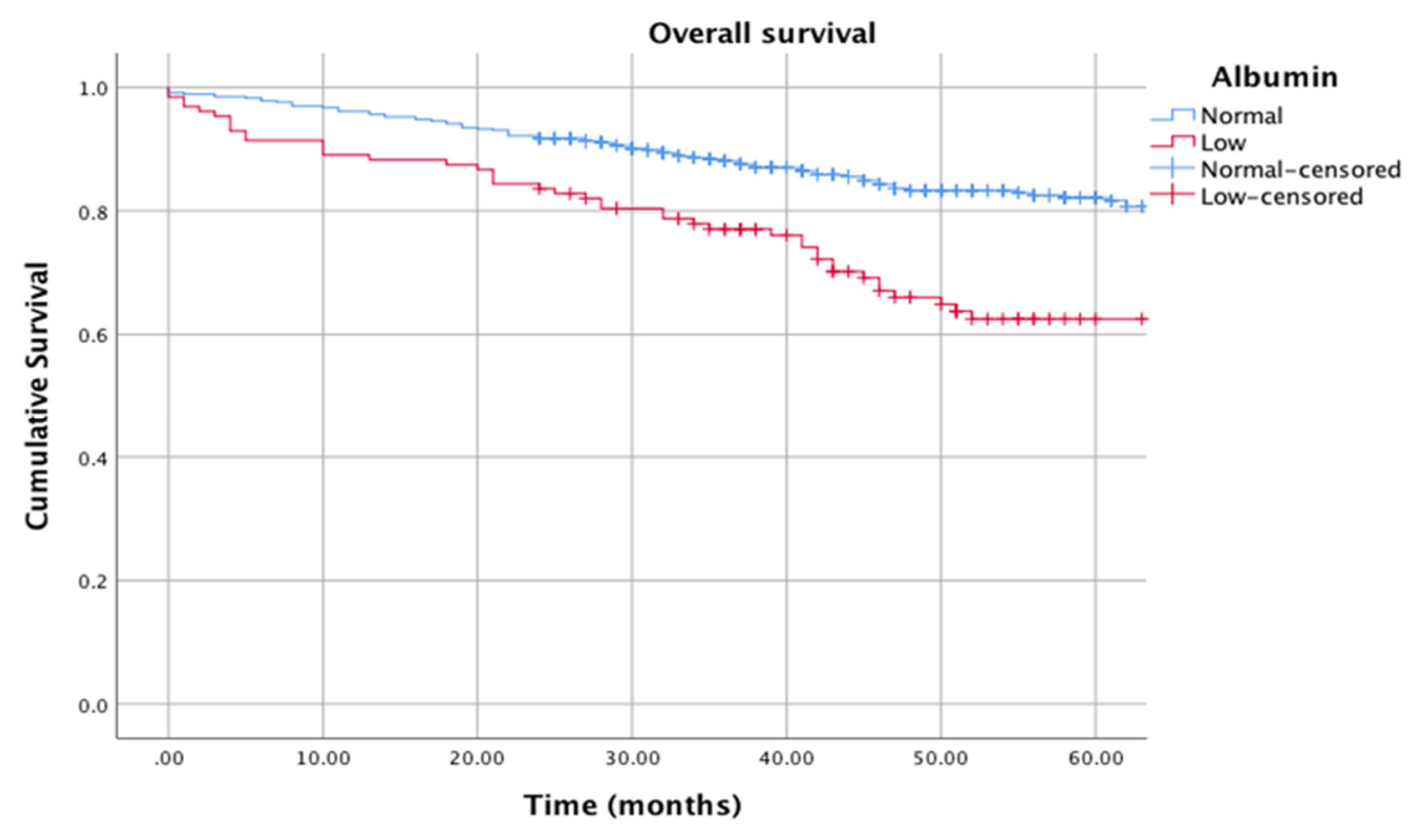

Figure 4. Kaplan-Meier curves showing the relationship between albumin (normal: $>35 \mathrm{~g} / \mathrm{L}$ vs. low: $<35 \mathrm{~g} / \mathrm{L}$ ) and overall survival (in the absence of a systemic inflammatory response, $\mathrm{CRP}<10 \mathrm{mg} / \mathrm{L}$ ). $n=589$. Median follow-up: 38 months. $\log$ rank $p=0.001$.

\section{Discussion}

The results of the present study show that hypoalbuminemia $(<35 \mathrm{~g} / \mathrm{L})$ was associated with a number of factors including older age, anemia, greater comorbidity and more advanced tumor stage. Also, hypoalbuminemia was associated with nutritional risk as evidenced by MUST and body composition analysis. Furthermore, hypoalbuminemia was consistently associated with measurements 
of the systemic inflammatory response. Finally, hypoalbuminemia was associated with overall survival and cancer-specific survival independent of measurements of the systemic inflammatory response. Therefore, hypoalbuminemia objectively reflects both nutritional and inflammatory status and contributes prognostic value to markers of the systemic inflammatory response.

In the present study of patients with operable colorectal cancer, approximately $30 \%$ had hypoalbuminemia, $25 \%$ had evidence of a systemic inflammatory response and 50\% had a low SMI prior to surgery. Therefore, even in early stage cancer, there is evidence of hypoalbuminemia, systemic inflammation and sarcopenia. Whilst the association between albumin and the systemic inflammatory response has been shown repeatedly in both acute and chronic inflammation $[25,26]$, there is limited data on the association between albumin and body composition. With reference to hypoalbuminemia and low SMI, the present results are consistent with two small studies. Low muscle mass was associated with lower serum albumin concentration when body composition was measured using dual-energy X-ray absorptiometry in the elderly [27]. Similarly, there was an association between albumin concentrations and lower lean tissue as measured using a total body potassium scanner [28].

The basis of the association of hypoalbuminemia and low SMI is not clear. However, it might reflect the widespread association between the chronic activation of the systemic inflammatory response and catabolic loss of muscle protein in patients with cancer. Indeed, it has been recognized that proinflammatory cytokines such as interleukin-6 (IL-6), interleukin-1 (IL-1), tumor necrosis factor (TNF) and growth factor (GF), released as part of the systemic inflammatory response, have a profound catabolic effect on host tissue metabolism. For example, IL-6 stimulates liver production of CRP and other acute phase proteins, thus increasing the demand for amino acids against a background of limited intake. In particular, low circulating glutamine concentrations (the most abundant amino acid in plasma) have been reported to be associated with hypoalbuminemia, a low SMI (major source of glutamine) and poorer survival in patients with colorectal cancer [29-32]. Therefore, it would appear the most abundant circulating amino acid (glutamine), circulating protein (albumin) and body protein are linked in a chronic systemic inflammatory response and that albumin reflects the inter-organ amino acid flux during the chronic systemic inflammatory response.

The combination of C-reactive protein and albumin, termed the Glasgow Prognostic Score (GPS), has been reported to be informative of the nutritional risk of patients with cancer [33] and has been used successfully to predict survival in patients with a variety of common solid tumors, both in the operable [10] and advanced inoperable setting [9]. Therefore, taken together, the literature points to close linkage of nutritional risk and the systemic inflammatory response in patients with cancer and indicates that if one is measured, then the other should be measured also [7].

There are striking parallels with the work carried out examining micronutrient status in health and disease [34,35]. For example, there is now recognition that iron status should be interpreted in the context of inflammatory status [36]. Indeed, the combination of C-reactive protein and albumin has been used for this purpose. This perhaps points to the fundamental linkage of metabolism and inflammation in patients with cancer as there would appear to be an intimate evolutionary link between immune and metabolic responses in all mammalian cells, so called immunometabolism [37].

The implications of the present observations are important. It has become clear that the systemic inflammatory response is an important etiologic factor in the development of cancer cachexia [38,39]. However, the presence of albumin is not included in the criteria. Given the present and recent results from Silva et al. [33] it is clear that hypoalbuminemia in the presence of a systemic inflammatory response offers readily available additional insight into body composition and the likely outcome of the patient with operable colorectal cancer.

The main limitation of the present study was that it was a retrospective study of patients in a single institution. In particular, only patients with available CT scans were included in the analysis. However, given that a CT scan was part of the routine workup for operable colorectal cancer, less than $10 \%$ of patients had missing CT scans. In addition, the measurements were taken at one set point in time (for colorectal cancer staging) and therefore is a "snapshot" of the patient's pathway and neither the 
point of onset of nutritional decline or changes in body composition (in particular skeletal muscle) are known. Therefore, there may be some selection bias in the present study. However, this study is, to our knowledge, the first to examine the association between a preoperative albumin, a nutritional risk tool (MUST), body composition, and systemic inflammation in a large number of patients undergoing surgery for primary operable cancer.

\section{Conclusions}

In summary, hypoalbuminemia was associated with greater nutritional risk, anemia, low skeletal muscle mass, low skeletal muscle density and the activation of the systemic inflammatory response. Moreover, it can provide useful independent prognostic value in patients with colorectal cancer.

Author Contributions: Conceptualization, D.C.M., A.S.A. and C.A.E.; methodology, A.S.A., D.C.M.; software, A.S.A. and R.D.D.; validation, A.S.A. and R.D.D.; formal analysis A.S.A. and D.C.M.; investigation, A.S.A. and D.C.M.; resources, A.S.A., D.C.M., C.A.E. and R.D.D.; data curation, A.S.A., D.C.M., and R.D.D.; writing - original draft preparation, A.S.A.; writing-review and editing, A.S.A., D.C.M. and C.A.E.; visualization A.S.A., D.C.M. and C.A.E.; supervision, D.C.M. and C.A.E.; project administration D.C.M. and C.A.E.; funding acquisition, A.S.A. All authors have read and agreed to the published version of the manuscript.

Funding: This study was funded by Saudi education sponsors, King Abdulaziz university, Faculty of Applied Medical Science, Department of Clinical Nutrition, Jeddah, Saudi Arabia.

Conflicts of Interest: The authors declare no conflict of interest.

\section{References}

1. Weaving, G.; Batstone, G.F.; Jones, R.G. Age and sex variation in serum albumin concentration: An observational study. Ann. Clin. Biochem. 2016, 53, 106-111. [CrossRef] [PubMed]

2. Christian, P.M.; Arturo, J.R.-D.; Deepansh, D.; Praful, R.; Akshay, S.; Julian, H.; Felix, K.H.C.; Adam, S.K.; Stuart, R.L.; Maxine, S.; et al. The association of hypoalbuminemia with early perioperative outcomes-A comprehensive assessment across 16 major procedures. Am. J. Surg. 2017, 214, 871-883.

3. McMillan, D. Systemic inflammation, nutritional status and survival in patients with cancer. Curr. Opin. Clin. Nutr. Metab. Care 2009, 12, 223-226. [CrossRef] [PubMed]

4. Jensen, G.L.; Mirtallo, J.M.; Compher, C.; Dhaliwal, R.; Forbes, A.; Grijalba, R.F.; Hardy, G.; Kondrup, J.; Labadarios, D.; Nyulasi, I.; et al. Adult starvation and disease-related malnutrition: A proposal for etiology-based diagnosis in the clinical practice setting from the International Consensus Guideline Committee. Clin. Nutr. 2010, 29, 151-153. [CrossRef] [PubMed]

5. Marcason, W. Should Albumin and Prealbumin Be Used as Indicators for Malnutrition? J. Acad. Nutr. Diet. 2017, 117, 1144. [CrossRef] [PubMed]

6. Soeters, P.B.; Wolfe, R.R.; Shenkin, A. Hypoalbuminemia: Pathogenesis and Clinical Significance. J. Parenter. Enter. Nutr. 2018, 43, 181-193. [CrossRef]

7. Arends, J.; Baracos, V.; Bertz, H.; Bozzetti, F.; Calder, P.C.; Deutz, N.E.P.; Erickson, N.; Laviano, A.; Lisanti, M.; Lobo, D.N.; et al. ESPEN expert group recommendations for action against cancer-related malnutrition. Clin. Nutr. 2017, 36, 1187-1196. [CrossRef]

8. McMillan, D.C. The systemic inflammation-based Glasgow Prognostic Score: A decade of experience in patients with cancer. Cancer Treat. Rev. 2013, 39, 534-540. [CrossRef] [PubMed]

9. Dolan, R.D.; Lim, J.E.K.; McSorley, S.T.; Horgan, P.G.; McMillan, D.C. The role of the systemic inflammatory response in predicting outcomes in patients with operable cancer: Systematic review and meta-analysis. Sci. Rep. 2017, 7, 16717. [CrossRef]

10. Dolan, R.D.; Laird, B.J.; Horgan, P.G.; McMillan, D.C. The prognostic value of the systemic inflammatory response in randomised clinical trials in cancer: A systematic review. Crit. Rev. Oncol. 2018, 132, 130-137. [CrossRef]

11. Feliciano, E.M.C.; Chen, W.Y. Clinical implications of low skeletal muscle mass in early-stage breast and colorectal cancer. Proc. Nutr. Soc. 2018, 77, 382-387. [CrossRef] [PubMed]

12. American Society of Anesthesiologists Martinench, A. American Society of Anesthesiologists: ASA Physical Status Classification System. 2014. Available online: https://www.med.ohio.gov/Portals/0/Resources/asaphysical-status-classification-system.pdf?ver=2019-04-02-083458-100 (accessed on 21 July 2020). 
13. Chan, M.; WHO. Haemoglobin Concentrations for the Diagnosis of Anaemia and Assessment of Severity; World Health Organization: Geneva, Switzerland, 2011.

14. Elia, M. The 'Must' Report Nutritional Screening of Adults: A Multidisciplinary Responsibility Executive Summary Section A: Screening for Malnutrition: A Multidisciplinary Responsibility; MAG: Redditch, UK, 2003; Available online: https://www.bapen.org.uk/pdfs/must/must-report.pdf (accessed on 21 July 2020).

15. ImageJ, Version 1.47; [software]. National Institutes of Health, 1997-2018. Available online: rsbweb.nih.gov (accessed on 3 July 2017).

16. Caan, B.J.; Meyerhardt, J.A.; Kroenke, C.H.; Alexeeff, S.; Xiao, J.; Weltzien, E.; Feliciano, E.M.C.; Castillo, A.L.; Quesenberry, C.P.; Kwan, M.L.; et al. Explaining the Obesity Paradox: The Association between Body Composition and Colorectal Cancer Survival (C-SCANS Study). Cancer Epidemiol. Biomark. Prev. 2017, 26, 1008-1015. [CrossRef] [PubMed]

17. Martin, L.; Birdsell, L.; Macdonald, N.; Reiman, T.; Clandinin, M.T.; McCargar, L.J.; Murphy, R.; Ghosh, S.; Sawyer, M.B.; Vickie, E.B. Cancer Cachexia in the Age of Obesity: Skeletal Muscle Depletion Is a Powerful Prognostic Factor, Independent of Body Mass Index. J. Clin. Oncol. 2013, 31, 1539-1547. [CrossRef] [PubMed]

18. Doyle, S.L.; Bennett, A.M.; Donohoe, C.L.; Mongan, A.M.; Howard, J.M.; Lithander, F.E.; Pidgeon, G.P.; Reynolds, J.V.; Lysaght, J. Establishing computed tomography-defined visceral fat area thresholds for use in obesity-related cancer research. Nutr. Res. 2013,33, 171-179. [CrossRef]

19. Ebadi, M.; Martin, L.; Ghosh, S.; Field, C.J.; Lehner, R.; Vickie, E.B.; Mazurak, V.C. Subcutaneous adiposity is an independent predictor of mortality in cancer patients. Br. J. Cancer 2017, 117, 148-155. [CrossRef] [PubMed]

20. Riesco, A. Five-year cancer cure: Relation to total amount of peripheral lymphocytes and neutrophils. Cancer 1970, 25, 135-140. [CrossRef]

21. Bruckner, H.W. Absolute granulocyte, lymphocyte, and moncyte counts. Useful determinants of prognosis for patients with metastatic cancer of the stomach. JAMA 1982, 247, 1004-1006. [CrossRef]

22. Maltoni, M.; Caraceni, A.; Brunelli, C.; Broeckaert, B.; Christakis, N.; Eychmueller, S.; Glare, P.; Nabal, M.; Viganò, A.; Larkin, P.; et al. Prognostic Factors in Advanced Cancer Patients: Evidence-Based Clinical Recommendations-A Study by the Steering Committee of the European Association for Palliative Care. J. Clin. Oncol. 2005, 23, 6240-6248. [CrossRef]

23. Hauser, C.A.; Stockler, M.R.; Tattersall, M.H.N. Prognostic factors in patients with recently diagnosed incurable cancer: A systematic review. Support Care Cancer 2006, 14, 999-1011. [CrossRef]

24. IBM SPSS Statistics for Windows, Version 24.0; IBM Corp: Armonk, NY, USA, 2016.

25. Gabay, C.; Kushner, I. Acute-Phase Proteins and Other Systemic Responses to Inflammation. N. Engl. J. Med. 1999, 340, 448-454. [CrossRef]

26. McMillan, D.C.; Elahi, M.M.; Sattar, N.; Angerson, W.J.; Johnstone, J.; McArdle, C.S. Measurement of the systemic inflammatory response predicts cancer-specific and non-cancer survival in patients with cancer. Nutr. Cancer. 2001, 41, 64-69. [CrossRef] [PubMed]

27. Visser, M.; Kritchevsky, S.B.; Newman, A.B.; Goodpaster, B.H.; Tylavsky, F.A.; Nevitt, M.C.; Harris, T.B. Lower serum albumin concentration and change in muscle mass: The Health, Aging and Body Composition Study. Am. J. Clin. Nutr. 2005, 82, 531-537.

28. McMillan, D.C.; Watson, W.S.; O'Gorman, P.; Preston, T.; Scott, H.R.; McArdle, C.S. Albumin Concentrations Are Primarily Determined by the Body Cell Mass and the Systemic Inflammatory Response in Cancer Patients With Weight Loss. Nutr. Cancer 2001, 39, 210-213. [CrossRef]

29. Don, B.; Kaysen, G.A. POOR NUTRITIONAL STATUS AND INFLAMMATION: Serum Albumin: Relationship to Inflammation and Nutrition. Semin. Dial. 2004, 17, 432-437. [CrossRef] [PubMed]

30. Hensley, C.T.; Wasti, A.T.; DeBerardinis, R.J. Glutamine and cancer: Cell biology, physiology, and clinical opportunities. J. Clin. Investig. 2013, 123, 3678-3684. [CrossRef] [PubMed]

31. Tuomisto, A.; Mäkinen, M.J.; Väyrynen, J.P. Systemic inflammation in colorectal cancer: Underlying factors, effects, and prognostic significance. World J. Gastroenterol. 2019, 25, 4383-4404. [CrossRef]

32. Ling, H.H.; Pan, Y.-P.; Fan, C.-W.; Tseng, W.; Huang, J.-S.; Wu, C.-J.; Chou, W.-C.; Wang, C.-H.; Yeh, K.-Y.; Chang, P.-H. Clinical Significance of Serum Glutamine Level in Patients with Colorectal Cancer. Nutrients 2019, 11, 898. [CrossRef] [PubMed] 
33. Da Silva, G.A.; Wiegert, E.V.M.; Lima, L.C.; Oliveira, L.C. Clinical utility of the modified Glasgow Prognostic Score to classify cachexia in patients with advanced cancer in palliative care. Clin. Nutr. 2020, 39, 1587-1592. [CrossRef] [PubMed]

34. McMillan, D.C.; Maguire, D.; Talwar, D. Relationship between nutritional status and the systemic inflammatory response: Micronutrients. Proc. Nutr. Soc. 2018, 78, 56-67. [CrossRef]

35. McSorley, S.T.; Talwar, D.; McMillan, D.C. Comment on the Biomarkers Reflecting Inflammation and Nutritional Determinants of Anemia (BRINDA) project. Am. J. Clin. Nutr. 2018, 108, 204-205. [CrossRef]

36. McSorley, S.T.; Tham, A.; Jones, I.; Talwar, D.; McMillan, D.C. Regression Correction Equation to Adjust Serum Iron and Ferritin Concentrations Based on C-Reactive Protein and Albumin in Patients Receiving Primary and Secondary Care. J. Nutr. 2019, 149, 877-883. [CrossRef] [PubMed]

37. Hotamisligil, G.S. Foundations of Immunometabolism and Implications for Metabolic Health and Disease. Immunity 2017, 47, 406-420. [CrossRef] [PubMed]

38. Cederholm, T.; Barazzoni, R.; Austin, P.; Ballmer, P.E.; Biolo, G.; Bischoff, S.C.; Compher, C.; Correia, M.I.T.D.; Higashiguchi, T.; Holst, M.; et al. ESPEN guidelines on definitions and terminology of clinical nutrition. Clin. Nutr. 2016, 36, 49-64. [CrossRef] [PubMed]

39. Cederholm, T.; Jensen, G.L.; Correia, M.; Gonzalez, M.C.; Fukushima, R.; Higashiguchi, T.; Baptista, G.; Barazzoni, R.; Blaauw, R.; Coats, A.J.S.; et al. GLIM criteria for the diagnosis of malnutrition-A consensus report from the global clinical nutrition community. Clin. Nutr. 2019, 38, 1-9. [CrossRef]

(C) 2020 by the authors. Licensee MDPI, Basel, Switzerland. This article is an open access article distributed under the terms and conditions of the Creative Commons Attribution (CC BY) license (http://creativecommons.org/licenses/by/4.0/). 